Revue

Revue de l'histoire des religions

del'histoire des religions

2| 2013

Écrire dans les pratiques rituelles de la Méditerranée antique. Identités et autorités

\title{
Écrire dans les pratiques rituelles de la Méditerranée antique
}

Identités et autorités. Avant-propos

Nicole Belayche et Francesco Massa

\section{OpenEdition \\ Journals}

Édition électronique

URL : http://journals.openedition.org/rhr/8105

DOI : $10.4000 /$ rhr.8105

ISSN : 2105-2573

Éditeur

Armand Colin

\section{Édition imprimée}

Date de publication : 1 juin 2013

Pagination : 155-165

ISBN : 978-2-200-92864-3

ISSN : 0035-1423

Référence électronique

Nicole Belayche et Francesco Massa, «Écrire dans les pratiques rituelles de la Méditerranée antique », Revue de l'histoire des religions [En ligne], 2 | 2013, mis en ligne le 01 juin 2016, consulté le 22 septembre 2020. URL : http://journals.openedition.org/rhr/8105; DOI : https://doi.org/10.4000/rhr 8105 


\section{Écrire dans les pratiques rituelles de la Méditerranée antique Identités et autorités Avant-propos}

Les mystères ( $\tau \varepsilon \lambda \varepsilon \tau \alpha i ́)$ d'Andanie en Messénie, célébrés en l'honneur des Grandes Déesses (Déméter et Corè), offrent un raccourci «historique» pertinent du thème de ce dossier: examiner comment l'écriture et les écrits dans les pratiques rituelles antiques, principalement grecques et romaines, ont servi à la construction d'identités locales et/ou ethniques et, de façon corrélée, comment écriture et écrits ont été utilisés dans la fondation de discours ou de positions d'autorité, dans des sociétés pourtant sans livres révélés sur lesquels se créerait la religion et qui ont longtemps été réputées avoir été des sociétés de l'oralité1. Au livre IV de sa Périégèse, Pausanias rapporte que, après la défaite spartiate à Leuctres (en 371 avant notre ère), l'argien Épitélès (au nom prédestiné) fut informé en rêve de creuser en un lieu du mont Ithomè.

Il trouva une lamelle d'étain réduite à une très fine minceur: elle

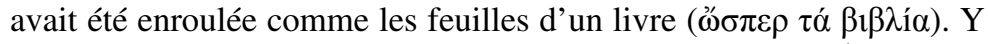

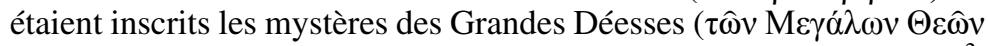

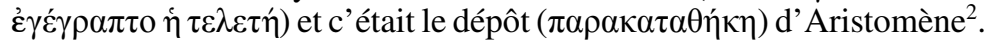

1. Ce projet est né d'un certain nombre de communications centrées sur l'Antiquité qui ont été présentées lors du Colloque International «Caratteri religiosi. Forme della parola scritta e pratiche rituali $\gg$ organisé à Modène (12-13 septembre 2011) par la Fondazione San Carlo, Modène et l'École Pratique des Hautes Études, Paris. Que les deux institutions, les organisateurs de la rencontre, et tous les participants trouvent ici l'expression de notre sincère reconnaissance.

2. Pausanias IV, 26, 8 (traduction Janick Auberger, Pausanias, Description de la Grèce, livre IV, Paris, 2005). Le schéma du récit d'invention peut être comparé, 
Le glorieux vaincu de la deuxième guerre de Messénie transmettait le legs identitaire d'un «talisman» capable «de recréer un État messénien à venir» et «de restaurer les mystères, en assurant la conservation des textes nécessaires aux rites $»^{3}$. Le Périégète signale aussi que «tous ceux qui appartenaient à la lignée des prêtres consignèrent [le contenu des lamelles] dans des livres

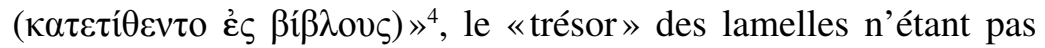
destiné à être manipulé lors des rituels. L'écrit assure de la pérennité du rituel porteur d'une référence identitaire. L'anecdote illustre bien la double fonction de l'écriture qui va être analysée: une fonction d'archivage des pratiques rituelles (parfois à partir d'un modèle réputé originel) $)^{5}$ qu'on retrouvera aussi à Rome, et une fonction d'autorité fondatrice d'une tradition cultuelle «nationale» et/ou familiale comme à Andanie. Dans les deux cas, le support même de l'écriture est différent: des rouleaux pour le moment rituel et un support plus précieux pour des documents de nature supérieure, comme l'étain, utilisé aussi dans des rituels dits magiques ou dans des rituels divinatoires ${ }^{6}$.

Les deux fonctions représentent, bien que différemment, des «lieux de mémoire» qui sont construits sur de l'appartenance et de la norme garantie par un pouvoir. Selon le Prométhée enchaîné d'Eschyle, l'art de la «combinaison des lettres» ( $\gamma \rho \alpha \mu \mu \alpha ́ \tau \omega v$

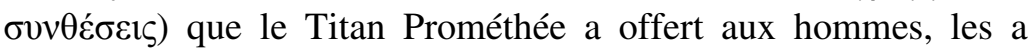
rendus capables de conserver la «mémoire de toutes choses $(\mu v \eta \dot{\eta} \mu$

mais dans un contexte différent (divinatoire et non cultuel), à la découverte des sorts de Préneste «gravés de caractères archaïques (insculptas priscarum litterarum)», Cicéron, De la divination II, 85.

3. Nadine Deshours, Les mystères d'Andanie. Étude d'épigraphie et d'histoire religieuse, Bordeaux (Ausonius, Scripta Antiqua 16), 2006, p. 191 \& 196-198. Voir désormais Laura Gawlinski éd., The Sacred Law of Andania: a New Text with Commentary, Berlin-Boston (Sozomena 11), 2012.

4. Pausanias IV, 27, 4. Sur la question des «écrits» à Andanie, Daniela Bonanno, «Memory Lost, Memory Regained. Considerations on the Recovery of Sacred Texts in Messenia and in Biblical Israel, a Comparison », dans Nicola Cusumano et al. éds., Memory and Religious Experience in the Greco-Roman World, Stuttgart, p. 63-80.

5. Cf. l'inscription du «règlement des mystères d'Andania», $I G \mathrm{~V} \mathrm{1,} \mathrm{1390,}$ 12-13: «Que les hieroi transmettent à leurs successeurs le coffret et les livres qu'a remis Mnasistratos» (traduction Nadine Deshours, Les mystères d'Andanie, p. 28 et commentaire p. 73-75); voir aussi ligne 84 les «écrits anciens ( $\delta i \grave{\alpha} \tau \hat{\omega} v$

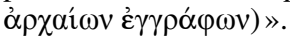

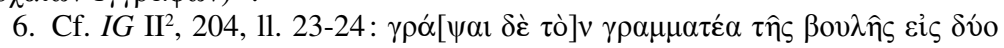

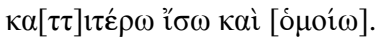


$\dot{\alpha} \pi \alpha ́ v \tau \omega v) »^{7}$. Mais les positions des auteurs anciens n'étaient pas unanimes. Au début du $\mathrm{IV}^{\mathrm{e}}$ siècle, le Socrate de Platon exprimait des réticences face aux effets de l'écriture, considérant qu'elle était un outil de remémoration (ínó $\mu \nu \eta \sigma ı)$ ), mais pas de mémoire ( $\mu v \eta \dot{\mu \eta})$, et qu'elle risquait «par négligence de la mémoire ( $\mu v \eta \dot{\mu \eta}$ s

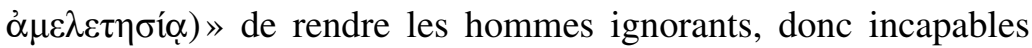
de progresser vers la sagesse ${ }^{8}$. Cette tension entre la position du philosophe attaché aux bienfaits de l'oralité pour acquérir un vrai savoir et la présence de l'écrit largement attestée dans les sociétés grecques et romaines invite à poser en préliminaires quelques données factuelles relatives à la réalité des écrits dans les pratiques religieuses.

\section{CONFIER LES PRATIQUES RELIGIEUSES À L'ÉCRIT?}

Depuis plus d'une génération, l'un des questionnements-phare de la recherche anthropologique concerne les relations entre oralité et literacy ${ }^{9}$ et leurs places respectives dans des sociétés majoritairement non alphabétisées ${ }^{10}$. Partant du constat que les religions polythéistes antiques n'avaient pas (sauf dans de petits cercles particuliers et plutôt tardifs) de livres ou de textes sacrés révélés du type de ceux des trois monothéismes réputés être des «religions du Livre» (ta Biblia en grec, al-Qur'an en arabe) ${ }^{11}$, leurs

7. Eschyle, Prométhée enchaîné 460-461.

8. Platon, Phèdre 275a-b. Sur la complexe fonction de l'écriture chez Platon, Mario Vegetti, «Dans l'ombre de Thot. Dynamiques de l'écriture chez Platon», dans Marcel Detienne éd., Les savoirs de l'écriture. En Grèce ancienne, Lille, 1992, p. 387-419.

9. Le néologisme français de «littéracie» (cf. Christine Barré-de Miniac, Catherine Brissaud \& Marielle Rispail, éds, La Littéracie. Conceptions théoriques et pratiques d'enseignement de la lecture-écriture, Paris, 2004) n'a guère été adopté pour les études sur l'Antiquité.

10. À partir de l'ouvrage fondateur de Jack Goody, The Domestication of the Savage Mind, Cambridge UP, 1977 (traduction française: La raison graphique. La domestication de la pensée sauvage, Paris, 1979), qui lie le développement de l'écriture à celui de la pensée logique, donc scientifique, cf. aussi Id., The Power of the Written Tradition, Washington DC, 2000 (traduction française Pouvoirs et savoirs de l'écrit, Paris, 2007).

11. Cf. Jan Bremmer, «From Holy Books to Holy Bible: an Itinerary from Ancient Greece to Modern Islam via Second Temple Judaism and Early Christianity», dans Mladen Popović éd., Authoritative Scriptures in Ancient Judaism, Leiden, 2010, p. 327-360. 
pratiques religieuses étaient rangées dans le camp de l'oralité, ce qui de surcroît s'accordait bien avec une certaine idée qu'on se faisait du ritualisme. Il est vrai que les divinités des panthéons classiques n'étaient pas représentées comme des «lettrés»: à quelques exceptions notables comme les Muses et Athéna ${ }^{12}$, elles ne sont presque jamais associées à l'écriture ou à des textes écrits. Lorsque dans les Grenouilles, Aristophane met en scène un Dionysos lecteur

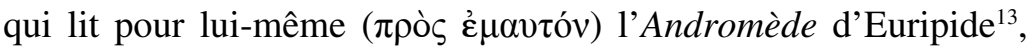
c'est précisément pour camper la condition «humanisée» du dieu du théâtre, descendu dans l'Hadès pour arbitrer un agôn comique entre Eschyle et Euripide.

L'absence de textes transmis par écrit et officiellement reconnus comme constituant un «canon» religieux n'empêchait pas que l'écrit fût présent dans les cérémonies cultuelles de la Méditerranée antique. Comme l'a montré Eric A. Havelock, dans un essai de 1982 devenu classique, c'est au $\mathrm{V}^{\mathrm{e}}$ siècle avant notre ère que la

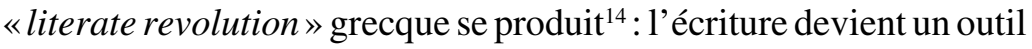
pour divers domaines (médecine, géométrie, histoire, géographie). Avant même cette époque dite classique, les cités grecques avaient commencé à écrire sur la pierre (ou d'autres supports) des textes à caractère législatif ou juridique ${ }^{15}$, dont ces «lois sacrées $»^{16}$ qui renferment des règlements, bien étudiés, sur la vie religieuse civique (calendriers festifs, sacrifices, offrandes, pratiques rituelles). À l'époque augustéenne, une cité d'Asie Mineure comme celle de

12. Cf. Albert Henrichs, «Writing Religion: Inscribed Texts, Ritual Authority and the Religious Discourse of the Polis », dans Harvey Yunis éd., Written Texts and the Rise of Literate Culture in Ancient Greece, Cambridge, 2003, p. 38-58.

13. Aristophane, Grenouilles 52-54.

14. Eric A. Havelock, The Literate Revolution in Greece and Its Cultural Consequences, Princeton, 1982 et The Muse Learns to Write: Reflections on Orality and Literacy from Antiquity to the Present, New Haven-London, 1986. Voir depuis, pour ne citer que trois travaux récents, Greg Woolf, «Literacy», The Cambridge Ancient History 11, 2000, p. 875-897; John F. A. Sawyer, Sacred Languages and Sacred Texts, London-New York, 1999; Mary Beard, «Writing and Religion» dans Sarah Iles Johnston éd., Ancient Religions, Cambridge, Mass.London, 2007, p. 127-138.

15. Voir, à ce propos, Marcel Detienne, «L'écriture et ses nouveaux objets intellectuels en Grèce», dans Id. éd., Les savoirs de l'écriture, p. 7-26.

16. Pour la discussion actuelle sur l'appellation impropre de «lois sacrées», Robert Parker, «What are Sacred Laws ? », dans Edward M. Harris, Lene Rubinstein éds., The Law and the Courts in Ancient Greece, London, 2004, p. 57-70 et Stella Georgoudi, "Comment régler les theia pragmata. Pour une étude de ce qu'on appelle "lois sacrées" », Métis n.s. 8, 2010, p. 39-54. 
Nysa conservait dans ses archives ( $\tau$ ò $\gamma \rho \alpha \mu \mu \alpha \tau \hat{\eta} o v)$ «les écrits

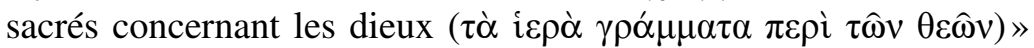
(des livres rituels probablement) et les attestations des privilèges du sanctuaire (asylie, atélie) ${ }^{17}$. En matière divinatoire, l'écriture fixait les réponses reçues d'Apollon dans ses sanctuaires spécialisés (par exemple Delphes, Claros et Didymes), et c'est par écrit, avec des lamelles, que les consultants de Dodone, cités ou particuliers, interrogeaient l'oracle de Zeus ${ }^{18}$. La fonction exemplaire que jouaient les oracles dans l'identité collective explique qu'ils aient très tôt été consignés par écrit et qu'il a dû exister, dès l'époque grecque classique, des collections ou anthologies d'oracles ${ }^{19}$, à commencer par celles que les sanctuaires eux-mêmes rassemblaient à fins d'illustration ${ }^{20}$. Dans Les Oiseaux, Aristophane tourne en ridicule l'autorité qui était censée découler des livres d'oracles brandis par un interprète et diseur d'oracles ( $\chi \rho \eta \sigma \mu \mathrm{o} \lambda$ ó$\left._{\mathrm{\gamma}} \varsigma\right)$ lors de la fondation de la nouvelle cité des oiseaux, située entre terre et $\operatorname{ciel}^{21}$.

Les sanctuaires pouvaient aussi graver dans la pierre d'autres éléments du rituel, comme les hymnes offerts aux divinités: à Delphes le Péan à Dionysos composé par Philodamos de Scarphée (c. 340 avant notre ère), qui chantait l'accueil réservé par Apollon à Dionysos lors des Théoxénies, était gravé le long de la Voie sacrée qui conduisait à l'oracle ${ }^{22}$. Les formes de dévotion personnelle ne furent pas moins conquises par la literacy. À partir de l'époque classique, les dévots confient régulièrement à la pierre le témoignage de leurs communications avec les dieux, multipliant dans les sanctuaires les textes d'offrandes et de satisfaction de

17. Syll. $.^{2}, 781,9-11$.

18. Cf. Herbert W. Parker, Donald E. W. Wormell, The Delphic Oracle: The History, 2. The oracular responses, Oxford, 1956; Joseph Fontenrose, Didyma. Apollo's Oracle, Cult and Companions, Berkeley-Los Angeles-London, 1988; Aude Busine, Paroles d'Apollon. Pratiques et traditions oraculaires dans l'Antiquité tardive (II ${ }^{e}-V I^{e}$ siècles), Leiden-Boston (RGRW 156), 2005 ; Éric Lhôte, Les lamelles oraculaires de Dodone, Genève, 2006.

19. Pour preuve la récurrence des formules dans des oracles différents, par ex. dans un oracle alphabétique de Tymbriada, Claude Brixhe \& René Hodot, L'Asie Mineure du Nord au Sud, Nancy, 1988, n 46.

20. Cf. Marie Delcourt, L'oracle de Delphes, Paris, 1981, p. 85-90.

21. Aristophane, Oiseaux $958-991$.

22. Cf. Miroslav Markovich, "Philodamus' Delphic Hymn to Dionysus», Zeitschrift für Papyrologie und Epigraphik 18, 1975, p. 167-168. 
leurs requêtes, bien avant l'explosion quantitative de la pratique épigraphique réputée caractériser le monde de l'Empire romain.

\section{Les Mémentos Rituels}

Pour Rome, les recherches sur les «archives de la piété $»^{23}$ ont d'abord permis de recenser la variété des écrits en relation avec les pratiques religieuses de l'État: depuis les livres inspirés et «fatidiques» du peuple romain - ces libri Sibyllini qu'Auguste reconstitua et installa dans le temple d'Apollon Palatin ${ }^{24}$ - jusqu' aux comptes rendus (commentarii) de fêtes exceptionnelles (comme les Ludi Saeculares) ou des activités annuelles des collèges de prêtres (Annales maximi des pontifes ou acta des Frères arvales) ${ }^{25}$. Ce faisant, elles ont permis de définir plus justement ce que la tradition historiographique a longtemps appelé «livres sacerdotaux»: ils servaient en réalité d' «outil du culte $»^{26}$ en conservant l'état du bon déroulement rituel - «la jurisprudence sacrée et les comptes rendus des rites célébrés » ${ }^{27}$, comme c'était déjà le cas à Andanie. Par-delà ces fonctions mémorielle et utilitaire, l'écrit s'avérait nécessaire dans le déroulement rituel lui-même afin de respecter les exigences d'un ritualisme rigoureux, de nature contractuelle, notamment dans

23. John Scheid, «Les archives de la piété. Réflexions sur les livres sacerdotaux», dans La mémoire perdue. À la recherche des archives oubliées, publiques et privées, de la Rome antique, Paris, 1994, p. 173-185.

24. Suétone, Vie d'Auguste 31 et Lactance, Institutions divines I, 6, 13.

25. Respectivement Bärbel Schnegg-Köhler, Die augusteichen Säkularspiele, München (Archiv für Religionsgeschichte 4), 2002; et John Scheid, Commentarii fratrum arvalium qui supersunt. Les copies épigraphiques des protocoles annuels de la confrérie arvale (21 av.-304 ap. J.-C.), Roma (Roma antica, 4), 1998. Cf. Richard Gordon, «Religion and Writing», dans Mary Beard, John North éds., Pagan Priests. Religion and Power in the Ancient world, London, 1990, p. 184191.

26. John Scheid, «L'écrit et l'écriture dans la religion romaine: mythe et réalité», dans Brigitte Gratien, Roger Hanoune éds., Lire l'écrit. Textes, archives, bibliothèques dans l'Antiquité, Ateliers. Cahiers de la maison de la recherche 12, 1997, p. 99-108 (107 pour la citation).

27. John Scheid, «"Livres" sacerdotaux et érudition: l'exemple des chapelles des Argées», dans Christophe Batsch, Ulrike Egelhaaf-Gaiser, Ruth Stepper éds., Zwischen Krise und Alltag. Antike Religionen im Mittelmeerraum. Conflit et normalité. Religions anciennes dans l'espace méditerranéen, Stuttgart (PAwB 1), 1999, p. 161. 
le prononcé des adresses précatoires ${ }^{28}$. Il fallait «lire d'abord la formule d'après le texte (de scripto praeire) ${ }^{29}$, afin de prévenir tout vice de forme du rituel. Le rôle de l'écrit est apparu si indispensable au bon fonctionnement des rites publics et à la pérennité même de $l^{\prime} V r b s^{30}$ qu'on a pu penser que «in Roman times, religions would have become more and more bookish $»^{31}$.

Ces mémentos rituels, porteurs d'une tradition et contrôlés et utilisés par des autorités sacerdotales et/ou civiques ${ }^{32}$, donnaient un statut d'autorité à l'écrit, mais pas un statut «consacré»-comme celui des biblia juifs qui déclenchait les sarcasmes d'un Juvénal bien romain dénonçant le comportement des Juifs «rabâchant et observant craintivement le fatras des lois juives transmises par Moïse dans un rouleau obscur $»^{33}$. La méfiance envers des cérémonies n'appartenant pas aux cultes civiques et dans lesquelles on utilisait des écrits n'était pas nouvelle. Euripide, Platon ou Démosthène, évoquant la présence de livres dans les cérémonies dionysiaques et orphiques, en profitaient pour critiquer et ridiculiser les cultes à la marge de la cités ${ }^{34}$. Pausanias rapporte que la cité arcadienne de Phénéos célébrait des cérémonies éleusiniennes. Lors de l'initiation

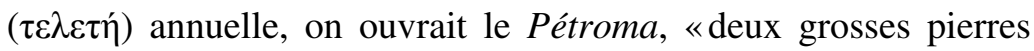
ajustées ».

28. Un article fondateur a été celui de Mary Beard, «Writing and religion: Ancient Literacy and the function of the written word in Roman religion », dans John H. Humphrey éd., Literacy in the Roman World (JRA Suppl. series 3), Ann Arbor, 1991, p. 35-58.

29. Pline, Histoire naturelle XXVIII, 3, 11; cf. Emmanuelle Valette-Cagnac, La lecture à Rome. Rites et pratiques, Paris, 1997, p. 254-280.

30. Cf. la dernière consultation des livres Sibyllins en 407 de notre ère, Claudien, Guerre gétique 230-232 et Palladius, Histoire lausiaque 119.

31. André P.M.H. Lardinois, Josine H. Blok, Marc G.M. Van der Poel éds., Sacred Words: Orality, Literacy, and Religion ( $8^{\text {th }}$ Intern. Conference on Orality and Literacy in the Ancient World, Netherlands), Leiden (Mnemosyne Suppl. 332), 2008, p. 2.

32. Cf. John North, «The Books of the Pontifices», dans Claudia Moatti éd., La mémoire perdue. Recherches sur l'administration romaine, Rome (Collection de l'École française de Rome 243), 1998, p. 45-63.

33. Juvénal, Satires XIV, 102: Iudaicum ediscunt et seruant ac metuunt ius, I tradidit arcano quodcumque uolumine Moyses (traduction Oliviers Sers, Paris, 2002, légèrement modifiée).

34. Cf. Euripide, Hippolyte 948-954 et Alceste 966-969; Platon, République II, 364c-365a et Démosthène, Discours XVIII, 259 (pour ces passages, voir l'article de Francesco Massa dans ce dossier). Sur l'écriture dans les cultes à mystères, cf. Walter Burkert, Les cultes à mystères dans l'Antiquité, Paris, 1992 [Harvard, 1987], p. 64-65. 
Après y avoir pris les écrits $(\gamma \rho \alpha ́ \mu \mu \alpha \tau \alpha)$ se rapportant à l'initiation

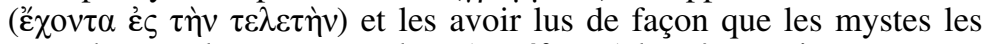
entendent, on les remet en place ( $\alpha \tau \tau \dot{\varepsilon} \theta \varepsilon v \tau o)$ la même nuit.

Et il poursuit:

Je sais que la plupart des gens de Phénéos jurent par le Pétroma

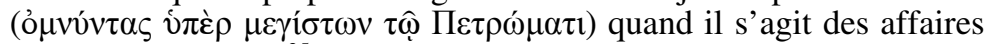
les plus importantes ${ }^{35}$.

Dans ces contextes cultuels particuliers, le statut des «écritures rituelles » donne une valeur opératoire et paradigmatique à l'écrit et l'écriture est lestée d'une valeur sacrée en soi ${ }^{36}$. À Rome, pendant la crise de la Seconde Guerre Punique (213-212 avant notre ère), le Sénat a vite mesuré le danger politique de l'invasion de formes religieuses, en grande partie d'origine étrangère, qui utilisaient des écrits. Il chargea le préteur Marcus Aemilius de retirer de la circulation les «recueils de prophéties et de prières ou de rituels de sacrifice » (libros uaticinos precationesue aut artem sacrificandi conscriptam $)^{37}$. Cinq siècles plus tard, sous l'empereur Valens, des oracles de pouvoir annonçant la mort violente de l'empereur et le désastre des Goths sortirent des murs sous la forme d'inscriptions anonymes quand on démolit la muraille de Chalcédoine ${ }^{38}$.

La place, réelle, de l'écrit dans les pratiques rituelles anciennes a suscité d'autant plus de réactions, voire de réserves, qu'elle assumait des fonctions d'identités et d'autorités que les cités ou États souhaitaient contrôler.

\section{UN PARCOURS ENTRE IDENTITÉS ET AUTORITÉS}

En examinant des contextes historiques précis - depuis la Sicile et la Grèce classiques jusqu'à la Méditerranée multi-religieuse d'époque romaine (Égypte, Anatolie, Proche-Orient) -, et en croisant diverses sources (inscriptions, papyrus, textes littéraires et images), les contributions ici rassemblées déclinent, chacune

35. Pausanias, Périégèse VIII, 15, 2.

36. Renée Koch Piettre, Christophe Batsch, «Avant-propos», Cahiers Mondes anciens 1, 2010, mis en ligne le 19 janvier 2010 (http://mondesanciens.revues.org/ index 128.html).

37. Tite-Live, Histoire romaine XXV, 1, 12.

38. Ammien Marcellin XXXI, 1, 4. 
dans son registre, comment écrits et actes d'écriture concourent à l'expression de discours identitaires et de positions d'autorité.

Le dossier s'ouvre sur la fonction jouée par l'écriture dans la construction d'identités qui se réalise dans des «lieux de mémoire» et de tradition. En s'attachant au sanctuaire des dieux Paliques, fondé dans la Sicile du $\mathrm{I}^{\text {er }}$ millénaire, Nicola Cusumano analyse le rôle que joue l'écriture dans la définition d'une ethnicité. Même si les témoignages littéraires insistent sur la nature «indigène» des Paliques, en s'appuyant notamment sur le serment ordalique, sorte de lieu de pouvoir, l'auteur établit que la présence de l'écriture rituelle a pénétré dans les cultures locales par le biais de la colonisation grecque. Cette conclusion lui permet d'engager la réflexion sur les processus de contact et les changements identitaires que l'écriture provoque. En se fondant également sur une documentation épigraphique, Anne-Rose HošEK étudie le cas de Deir el-Qalaa, sanctuaire extra-urbain de la colonie romaine de Berytus en Syrie. Une analyse très serrée des manières d'exprimer les noms des dieux en latin et en grec met en évidence des décalages entre les façons de les dire et conduit à la conclusion que l'écriture en grec ne joue que le rôle de passeur de culture. Le dialogue, lorsqu'il s'établit, met en contact des traits culturels portés par une langue sémitique (probablement parlée) et des traits culturels romains. Ces deux études montrent comment les processus de constitution identitaire, généralement encadrés, se déploient entre autres au moyen de textes écrits ou de l'écrit, qui marquent, et parfois inventent, les frontières de l'identité. Lorsqu'on croise identités culturelles et cultuelles, la pratique de l'écriture se révèle être un marqueur particulier des rituels de type initiatique. Les cultes à mystères s'en servent souvent pour valider, et procéder à, leurs déroulements rituels, comme on l'a vu à Phanéos d'Arcadie. Pourtant, la présence des textes ne s'y limite pas à leur fonction pragmatique, ce que montrent bien les représentations imagées de rouleaux et tablettes dans les cérémonies rituelles, qui n'évoquent pas seulement le contenu de hieroi logoi ou de prescriptions cultuelles. Représenter les objets de l'écrit est aussi un langage iconographique pour dire un savoir culturel ou religieux et le respect de l'ordo liturgique, pour affirmer la participation à un monde ordonné. En analysant des passages littéraires sur papyrus de l'Égypte gréco-romaine et des représentations figurées relatifs aux rituels initiatiques dionysiaques, Francesco Massa s'interroge 
sur la présence et le rôle de textes écrits dans ce culte. Même si la documentation est restreinte, elle atteste de la présence de rouleaux ou de tablettes qui contenaient à la fois des références aux récits mythiques et des éléments utiles au déroulement des pratiques rituelles bachiques. On peut valider cette conclusion en remontant le temps. L'analyse archéologique et historique menée par Valeria Piano sur le papyrus de Derveni, dans la Macédoine de la fin du $\mathrm{IV}^{\mathrm{e}}$-début du $\mathrm{III}^{\mathrm{e}}$ siècle avant notre ère, tire profit à la fois d'une comparaison avec les rites funéraires de la région et de certains points exégétiques et philosophiques du texte. Depuis l'Antiquité, les textes dits orphiques ont été associés à la pratique de l'écriture ${ }^{39}$. Le papyrus de Derveni, qui conserve une parole attribuée à Orphée et le commentaire philosophique et religieux d'un auteur anonyme, offre un témoin «historique» de l'usage de l'écriture, qui sert ici aussi de psychopompe pour le défunt, fondé sur le discours sacré.

Le rôle de l'écriture dans la diversité des constructions identitaires qui viennent d'être exposées a déjà mis en évidence combien il s'appuyait aussi sur une valeur d'autorité, issue soit du media lui-même soit de son «auteur». C'est cette valeur de «pouvoir» et d'«autorité» qui est au centre des deux dernières contributions, puisque l'écriture est aussi un outil, à la fois objectivé et durable, sur lequel se construit le pouvoir. Dans cette perspective, l'attention n'a pas été mise sur la forme de pouvoir et de contrôle exercée par des autorités (politiques et/ou religieuses) sur les textes écrits, mais sur les formes de pouvoir, humains et divins, qui s'établissent par le recours à l'écrit. En s'attachant à une documentation originaire de l'Anatolie romaine rurale, Nicole BELAYCHE examine un ensemble de textes gravés connus sous l'appellation de «stèles de confession», datant pour l'essentiel des II $^{\mathrm{e}}$-milieu du $\mathrm{III}^{\mathrm{e}}$ siècles de notre ère. Ces textes, qui satisfont aux deux fonctions habituelles de l'épigraphie - fonction mémorielle et fonction publicitaire ou honorifique -, démontrent surtout que la mise par écrit, contrôlée par les prêtres et présentée comme indispensable, est un instrument de pouvoir. La substance des textes consiste en une exaltation des dieux, réclamée par les dieux eux-mêmes: c'est sur elle que

39. Cf. Claude Calame, «Pratiche orfiche della scrittura: itinerari iniziatici?», dans Giulio Guidorizzi, Marxiano Melotti éds., Orfeo e le sue metamorfosi. Mito, arte, poesia, Roma, 2005, p. 28-45. 
se pérennise leur «royauté» sur les communautés villageoises, donc le pouvoir des prêtres qui les servent. Dans un contexte fort différent, les rituels de la magie hébraïque nourris à des traditions culturelles variées et emboîtées expriment des rapports de pouvoir et d'autorité analogues, bien que plus complexes puisque des pouvoirs contraires s'affrontent. Emma АBATE procède à une mise en série de textes rituels d'origine juive destinés à des prophylaxies antidémoniaques, qui courent de l'époque hellénistique au Moyen Âge (les manuscrits de la Genizah du Caire, dont deux fragments inédits), en passant par les écrits de Qumrân et les papyrus magiques grecs. Analysant les pratiques d'exorcisme prescrites pour contrôler les démons, l'écriture s'y révèle un instrument puissant et efficace. La comparaison sur la longue durée met en évidence l'existence de schémas récurrents d'expulsion démoniaque dans une vaste zone de la Méditerranée orientale.

nicole.belayche@ephe.sorbonne.fr

f.massa@tin.it 